\title{
Orchestrating an ubiquitous learning situation about Cultural Heritage with Casual Learn
}

\author{
Anonymized \\ Anonymized
}

\begin{abstract}
Most of the existing ubiquitous situations to learn Cultural Heritage are not related to formal education, nor designed or assessed by a teacher. This paper presents a case study that analyzes the orchestration of a ubiquitous learning situation involving a teacher and 89 secondary-school students. In this situation, the students firstly proposed in their classroom descriptions of Cultural Heritage sites and tasks to be done when visiting them. Then, the students used Casual Learn to carry out the out-classroom proposed tasks. This case study allows us to illustrate how teachers can use Casual Learn to orchestrate ubiquitous learning situations to learn Cultural Heritage. In fact, in this learning situation, Casual Learn played a key role as it enabled to bridge in- and out-classroom activities across physical and virtual learning spaces.
\end{abstract}

Keywords: Ubiquitous learning, Cultural Heritage learning, case study, Mobile learning

\section{Introduction}

Cultural Heritage learning aims to offer a holistic and multidisciplinary view about past and present societies [23]. This view includes the artistic, architectonic, historical, ethnographic, technological, and economical aspects of these societies [14]. Cultural Heritage learning is included in the secondary-education curriculum in many countries, as it develops critical thinking, tolerance, student's self-identity, and promotes the preservation of historical monuments [11, 14]. Cultural Heritage learners highly benefit from active on-site learning: learners get a better understanding of a monument and its context when visiting it, than when studying it from a book or online site [11]. For this reason, schools traditionally organize trips to visit historical buildings or museums. However, culturally enriching field trips are not as common as they used to since they are expensive and logistically complex $[6,11]$.

Existing software tools to learn Cultural Heritage can contribute to alleviating that shortage of field trips. For instance, serious games [17] or virtual reality applications [6] are increasingly used for Cultural Heritage learning and, sometimes, as alternatives to school trips. However, these technologies cannot replace the experience of visiting the actual monument $[6,17]$. In that sense, mobile applications have also been developed for Cultural Heritage learning [4, 10, 22], mostly providing information about Cultural Heritage sites by making use of the 
geo-position of the user. This type of applications can trigger interesting opportunities for out-classroom learning but such opportunities are often completely disconnected from what is going on at formal education institutions (schools, high schools, etc.).

The seamless connection of in-classroom or online activities of formal learning contexts with out-classroom learning activities happening, e.g., near a Cultural Heritage site, is a key feature of the so-called Ubiquitous Learning (UL) [13]. UL underlines the importance of learner autonomy, continuity across contexts, and situational learning [13,19], an approach that several authors call seamless learning [16]. Also, UL encompasses across-spaces learning, characterized by the involvement of different physical and virtual learning spaces in the same learning situation [8].

An example of a mobile application that may support UL for Cultural Heritage is Casual Learn [1,2]. Casual Learn, designed and developed by the authors, makes use of semantic technologies and linked open data to define 10000 active learning tasks related to Cultural Heritage sites from the Spanish region of Castile and Leon. Such learning tasks were defined taking into account the official curriculum of the regional education system and were validated by secondary education teachers [3]. Casual Learn is already available in Google Play Store for downloading and installation ${ }^{1}$, and it has already been tested by a set of individual users. However, this paper goes a step forward and explores the implications of using Casual Learn in a real educational setting aimed at connecting in-classroom learning activities with out-classroom learning activities happening in the surroundings of Cultural Heritage sites.

More concretely, this paper presents the results of a study focused on the difficulties faced by a secondary-education teacher when designing and enacting an UL situation supported by Casual Learn. Such difficulties, faced by teachers when dealing with complex technology-enhanced learning settings, are studied, by the research community on technology-enhanced learning, under the umbrella of the so-called orchestration metaphor [9]. Therefore, this paper tackles the following issue: to what extent can teachers orchestrate Casual Learn as part of their teaching practice in a manageable way?

The rest of the paper is structured as follows: section 2 briefly introduces the state of the art of UL for learning Cultural Heritage. Section 3 provides further details of Casual Learn. Section 4 describes the methods used in our study, whose results are reported in section 5. Finally, section 6 summarizes the most important conclusions and the lines of future research.

\section{Ubiquitous learning for Cultural Heritage}

UL is typically defined as "using mobile technologies to facilitate learning", although the definition of "learning anywhere and at anytime" [13] is arguably more popular. The idea behind both definitions is that UL situations happen in

\footnotetext{
${ }^{1}$ https://casuallearnapp.gsic.uva.es
} 
different physical (e.g., a classroom or a museum) or virtual (a learning management system or a mobile app) spaces mediated by mobile devices $[13,19]$. UL promotes autonomous and active learning across different spaces and contexts. In many cases, UL situations bridge formal and informal learning contexts, where learning tasks of different formality may occur [8]. For example, in a History of Art course, after the teacher explains the Gothic art in the classroom (physical space in a formal context), she may ask her students to prepare an online document with a list of the main architectural features of a Gothic cathedral (virtual space within a formal context) that will be available if the students happen to be close to the Gothic cathedral of their city (physical space in an informal context). Then, some students may pass by the cathedral, and they may be asked to read the features written by their colleagues or do some exercises with their phones (virtual space in an informal context). Once back to the classroom, the teacher may organize a debate for the students to share their experience in the cathedral. Thus, this UL situation would combine the advantages of different spaces and would seamlessly relate formal and informal learning tasks [7].

All these UL benefits come to a price: using mobile technology to bridge learning across spaces may require a significant effort for the teacher. UL typically implies using new technological tools and platforms. Moreover, there are important pedagogical issues in the orchestration of UL situations, including their learning design [19] (e.g., how to define the sequence of activities that will be carried out, including how the tools will support them) and enactment [18] (e.g., scaffolding the students or solving their doubts). In the last few years, the research community is making a significant effort to overcome these issues, but few researchers explored them in the context of UL situations that bridge in-classroom and out-classroom learning [7].

Overcoming the aforementioned limitations of UL could be highly beneficial for Cultural Heritage learning situations: since school trips are scarce (especially in pandemic times [4]) [11], it is convenient to help the teacher to orchestrate learning situations that connect in-classroom activities with on-site activities that students may carry out in informal contexts. However, the potential of UL for the Cultural Heritage domain has not been explored in depth $[7,13,19]$. Some researchers reported UL situations either inside museums [22] or around the city [10]. But these are purely informal learning experiences, not related to formal learning or contexts. Furthermore, they do not promote active learning as the supporting technology limits its functionality to offering [10] or recommending [22] information about places to visit. Abril-López et al. [4] reported an UL situation for preservice teachers that includes activities in the classroom and in a local museum. While interesting, this is a highly structured learning activity for a specific outdoor context that makes limited use of UL technology. 


\section{Casual Learn}

Casual Learn $[1,2]$ is a mobile application to support UL situations to learn Cultural Heritage. It was designed in an iterative process that involved 13 teachers from five secondary schools of the Spanish region of Castile and Leon.
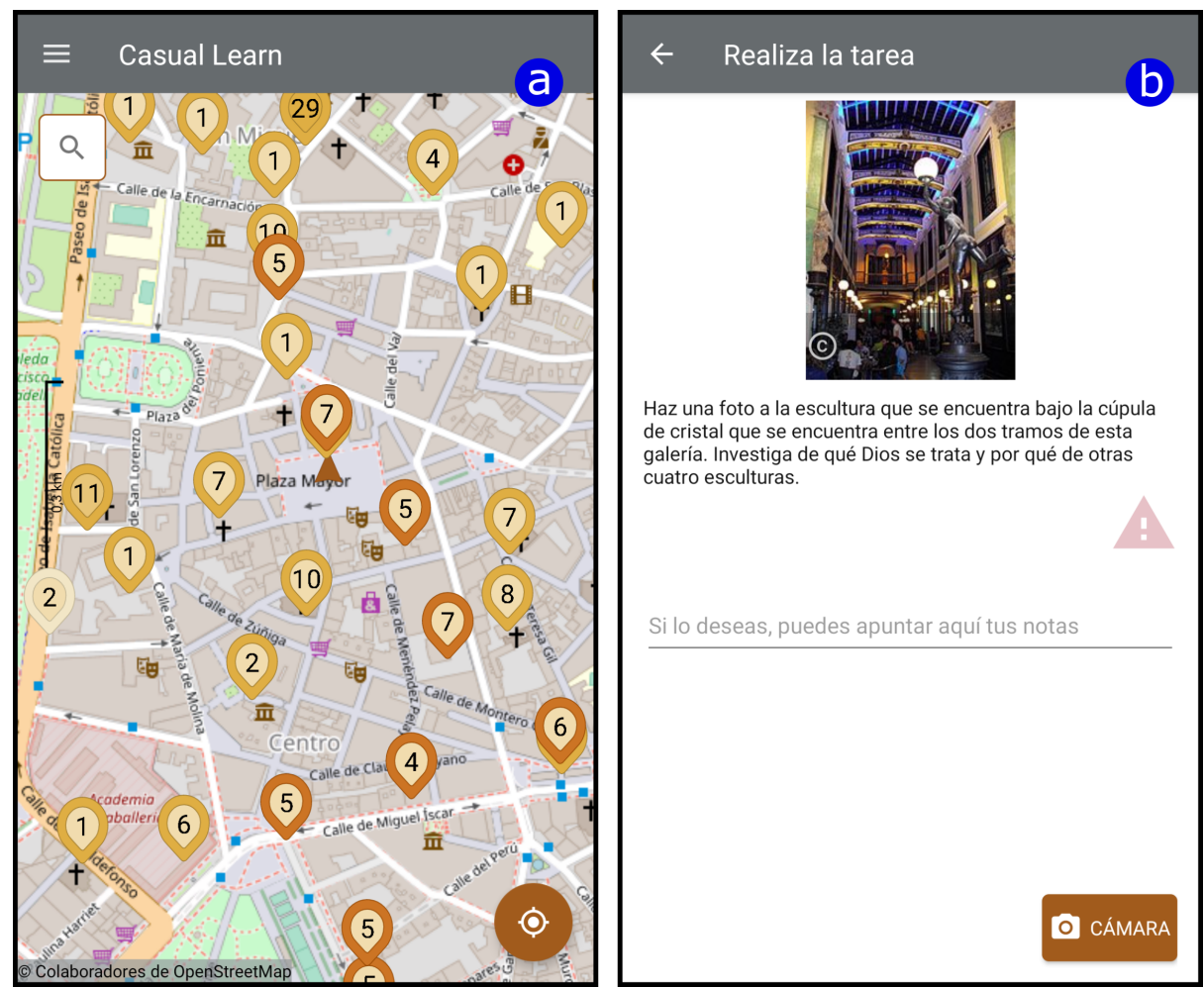

Fig. 1. Snapshots of the user interface of Casual Learn. a) view of the city center of Valladolid; icons represent Cultural Heritage sites where a number of tasks are located. b) a task description related to the "Pasaje Gutiérrez"; the textual task description is "Take a photograph of the sculpture located under the glass dome. Find out which god it is representing and why there are four other sculptures".

Casual Learn includes a set of tasks contextualized in Cultural Heritage sites that learners can browse in a map (see Figure 1a). Once the learner approaches a Cultural Heritage site, she can carry out the tasks related to it, which may entail reading a text, taking some photographs, recording a video, or answering some questions. For example, Figure 1b depicts a task where the student is asked to take a photograph of a sculpture and reflect on its meaning. The answers to the tasks can be reported in a portfolio and may also be shared in social networks (Instagram, Twitter, and Yammer) or learning environments (Microsoft Teams). 
As a differentiating characteristic, Casual Learn is based on Linked Data technology and counts with a dataset of more than 10000 tasks that were semiautomatically created out of Web Open Data [3]. All these tasks are related to Cultural Heritage sites of Castile and Leon. Additionally, Casual Learn recommends Cultural Heritage sites to approach to, and tasks to do, when learners are close to them. Thus, learners can use Casual Learn in an active mode (looking for tasks to do) and in a passive mode (getting recommendations). Casual Learn also enables the definition of collections of tasks (e.g., tasks related to a particular topic). As an example, Figure 1a shows two collections of tasks, that can be distinguished by the shade of color of the icons belonging to each one of them. Thus, learners can easily detect in which locations they can find tasks of their interest. More information about Casual Learn can be found in [1-3].

\section{Methods}

ISSUE: To what extent can teachers orchestrate Casual Learn as part of their teaching practices in a manageable way?

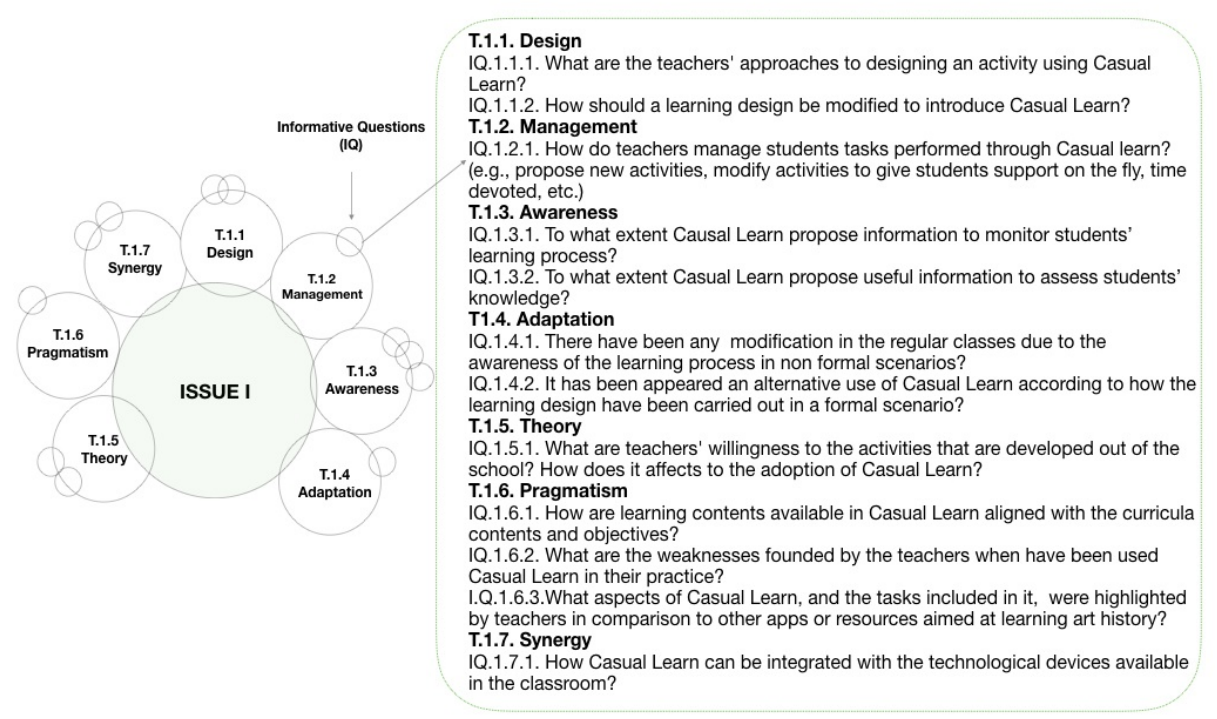

Fig. 2. Anticipatory data reduction schema showing research questions (RQ), issues (I), and topics $(\mathrm{T})$.

The main aim of this study is to explore the issue: to what extent can teachers orchestrate Casual Learn as part of their teaching practice in a manageable way? The study is framed within an interpretative research paradigm, which does not pursue statistically-significant results, rather aiming to a deep understanding of the particularity of the concrete phenomena under study [12]. During the 
evaluation design, we used an anticipatory data reduction process [15], using as its basis the $5+3$ aspects orchestration framework [20]. Figure 2 depicts this anticipatory data reduction schema.

\subsection{Research context}

This case study was carried out in a public secondary-education school located in a middle-class district of the city of Valladolid (Castile and Leon, Spain). The participants were a teacher of History with more than 20 years of teaching experience, and 89 students of the fourth grade of secondary education (15-16 years). The students belonged to four different classes.

History is a compulsory course for these students. The curriculum design proposed by the National Educational Law and the regional educational legislation establishes that the course of History in the fourth grade of secondary education should cover the XIXth and XXth Century History. One of the topics to address is the rise of the urban bourgeoisie during the XIXth Century. However, teachers are autonomous to define the academic program of their subjects -according to the school's values, objectives, and priorities- and the pedagogical techniques employed [21]. The secondary school where this case study was carried out recently included among its priorities the promotion of active, collaborative, and competence-based learning. However, neither the participant teacher nor the students had previous practical experience with these learning methodologies.

Valladolid has a vast number of XIXth Century Cultural Heritage sites related to the rise of the bourgeoisie in the city. The teacher used to take advantage of this fact by organizing a school trip every year in which they visited these sites with her students and a local tourist guide. This helped her students to better know their own city and relat its Cultural Heritage sites to the historical events studied in the classroom.

However, in the course 2020/2021, school trips and organized tourist visits were not allowed due to the restrictions related to the COVID-19 pandemic, although people could walk freely around the city of Valladolid, alone or in small groups. This opens the possibility that the students visit Cultural Heritage sites in Valladolid and use Casual Learn to carry out some tasks previously proposed by the teacher.

\subsection{Learning design}

The aim of the learning situation was to understand the rise of the bourgeoisie in the city of Valladolid, as well as the main events that happened in the city in the, so-called, Bienio Progresista (1854-1856). This topic had been explained by the teacher before the learning situation started. The UL design is depicted in Figure 3. The learning situation was planned to last for four weeks (from the 15th of February to the 14th of March 2021) and was divided into two phases:

During the first phase -planned for 14 days- the students proposed tasks to be done with Casual Learn. The teacher divided each of the four classes into four groups of four or five students. Each group was assigned a topic related to the 
general aim of the learning situation (culture, economy, politics, and society) and a set of four or five Cultural Heritage sites of Valladolid related to their topic (18 sites in total). The teacher provided each group with some basic bibliography that they were expected to extend. Then, learners should produce, for each Cultural Heritage site, an electronic document that contains a short description of the site, an extended description which may contain images and references, and a set of tasks to be done by their colleagues. Then, each group gave a short presentation to their classmates explaining the sites assigned to them.

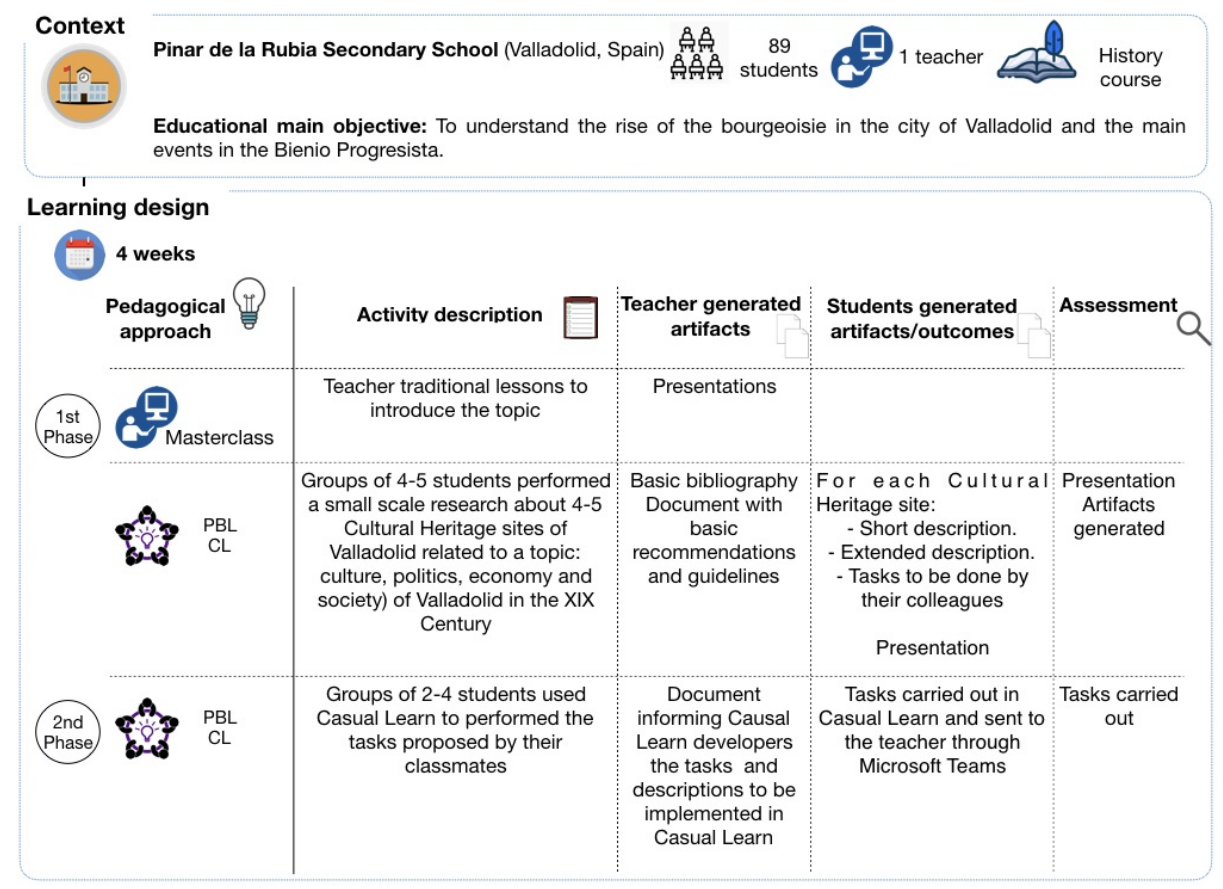

Fig. 3. Graphical depiction of the learning design.

Once the first phase was completed, the teacher collected and reviewed all the abstracts, descriptions, and tasks proposed by the students to be included in Casual Learn (this was planned for five days). As the current version of Casual Learn does not count with a data publication interface, it was the researchers who published all this information in Casual Learn. The tasks proposed by each class belonged to a different task collection, so learners could distinguish them; i.e., the 18 Cultural Heritage sites were the same for the four classes, but the tasks of each class were surrounded by different colors.

During the second phase -planned for the last ten days- the students used Casual Learn to carry out the tasks proposed by their classmates. They freely 
formed groups between two and four students. In their evenings or weekends they had to visit the 18 Cultural Heritage sites proposed by the teacher.

For each site, each group had to read the information offered and carry out all the tasks proposed by their classmates (i.e., those surrounded by the colour assigned to their class). They should also use Casual Learn to send their answers to the teacher via Microsoft Teams. Optionally, they could do other tasks proposed by Casual Learn (either tasks already existing in the application or tasks proposed by other classes) and also share their answers with their teacher.

The first phase of the learning situation was compulsory for all the students, while the second phase was optional (students could do other homework instead). The teacher assessed both phases of the activity using a rubric that she shared with her students before the first phase of the learning situation started. This rubric took into account both individual and team competencies. She evaluated the documents generated by the learners, their presentation to the rest of the class, and their behavior during the learning situation.

\subsection{Research design}

Four months before the case study took place, the authors of this paper gave a two-session seminar about Casual Learn to the teacher who participated, so that she could understand its functionality. Then, the teacher freely created the learning design, asking the authors if she had any doubt about Casual Learn.

We collected several data to analyze the learning situation. During its enactment, we were in close communication with the teacher but did not attend any of the sessions (due to the pandemic situation, only students, teachers, and school workers could legally get inside the school). We had an informal interview every week with the teacher while its enactment, so she reported the progress to us ([WI]). Once the learning situation finished, we had a semi-structured interview with the teacher $([\mathrm{I}])$. We collected some questionnaires from the teacher ([TQ]) and the students ([SQ]) asking for the Casual Learn's perceived usability and utility. We also collected several documents related to the learning situation: the learning design; the documents offered by the teacher to scaffold the students; the evaluation rubric; and all the documents created by the students (see Figure 3). Finally, we also analyzed Casual Learn's logs ([L]).

\section{$5 \quad$ Results}

\subsection{Enactment}

The learning situation was successfully carried out. However, several aspects needed to be changed from the original design [WI]. The most relevant change was its duration: the first phase took three weeks instead of two, and the second phase took 16 days instead of 10 . This second phase was also postponed for a few days because the students were busy with exams of other courses. Hence, the learning situation was not finished until the 6th of April. 
The students proposed tasks for Casual Learn during the first phase of the situation. However, the teacher had to scaffold them more than she had expected: she suggested to her students more material to read, and she proposed some questions to help their critical analysis. Nonetheless, the descriptions and tasks proposed by the four groups were very similar; according to the teacher this is because of their lack of critical analysis: "I thought that the students would look for other sources and they would find different viewpoints for the routes[...] But this was not the case: the routes were somehow repetitive" [I]. For this reason, the teacher decided to implement in Casual Learn only the tasks and descriptions proposed by two groups. Overall, 36 descriptions and 74 tasks were published in Casual Learn (see Figure 1b for an example of a task).

80 out of 89 students carried out the tasks with Casual Learn [SQ]. They were divided into 23 groups of three or four students each. They carried out 451 tasks in Casual Learn [L]: 222 tasks proposed by a group; 199 proposed by another group; and 30 tasks previously existing in Casual Learn and carried out by 13 groups of students. This shows that 13 groups not only carried out the compulsory tasks but also completed their learning by doing other tasks available in the application.

Each time a group of students carried out a task, they submitted it to the teacher via Microsoft Teams. This way, she was aware of the groups' progress. The teacher only needed to intervene once during this second phase of the situation: because of technical problems, Casual Learn was not available for two hours. The students contacted the teacher to know the reason, and she asked the authors, who explained the issue. Then, she notified the students.

Generally speaking, the students perceived that they learned during the situation [SQ]. Indeed, when comparing this situation to a traditional classroom, $59.74 \%$ of the students considered that they participated more actively while only $2.6 \%$ consider that they participated less actively; $79.22 \%$ agreed that it better contributed to improving their oral and written expression, while $20.78 \%$ disagreed; and $80.52 \%$ agreed that they reached a deeper knowledge about the topic, while $19.48 \%$ disagreed. However, some of them complained that the second phase of the situation was too long, as they had to visit 18 Cultural Heritage sites ("the activity was too long", "there were too many tasks to do" [SQ]).

The students were also asked about Casual Learn [SQ]. In a System Usability Scale (SUS) [5] the score obtained was 67.16 out of 100. Many of them pointed out that they liked the idea of visiting and learning about buildings of their own city that they did not know. Some of them also suggested us [SQ] to develop an iOS version of Casual Learn and others to explicitly implement "routes", so the application would guide users in the order that they should visit the Cultural Heritage sites.

\subsection{Orchestration}

This subsection reports the main findings obtained in the evaluation regarding the orchestration of the learning situation. The findings are organized following the topics defined in the anticipatory data reduction process (see Figure 2). 
Design: the evaluation showed that Casual Learn enabled the teacher to implement an UL design to learn Cultural Heritage. This design included collaborative in- and out-classroom across physical and virtual spaces.

During the interview, the teacher said that, before knowing Casual Learn, she had in mind to carry out a collaborative out-classroom activity. She wanted her students to follow the historical method in order to relate the concepts, events, and facts taught in the classroom to the Cultural Heritage sites of Valladolid. But, as she mentioned, she did not know how to design such an activity. Then, she saw Casual Learn as an opportunity that facilitates her design, as it can explicitly relate texts and tasks to Cultural Heritage sites: "Casual Learn arrives when I had in mind designing a project applying new technologies. I had seen and selected some texts and web pages. I knew how to assess it [and reflected about] how it would be for the students and teachers. Then Casual Learn arrives and when you showed it I thought it was ideal for this case. I saw that this is an application where you define a set of sites [...] and let the students learn in a more informal way. So I thought that it was good for the students, who are very motivated each time I ask them to do something with their phones in class. Hence, I thought this application was ideal for my case. [I]"

It is noteworthy that, in pandemic times, it is not possible to do school trips, as the teacher used to do with her students. In this sense, Casual Learn is a technology that enabled the implementation of a learning design that alleviated the impact of this restriction in the course she teaches.

Management: in this case study the teacher could manage the UL situation with almost no support from Casual Learn developers.

During the first phase of the situation, she had to further scaffold students offering them more sources and motivating them to deepen their analysis: "[many groups of students] took a couple of sources and wrote a brief summary out of them" [I]. Hence, she had to support their critical analysis and extend the duration of the activity.

During the second phase of the situation, the students used Casual Learn without the need for further support [WI]. They were autonomous when using Casual Learn on their own devices; indeed, they perceived it as a usable application [SQ]. There was only a group of students who asked the teacher for support because Casual Learn was not available at a certain moment.

Awareness: this evaluation shows that Casual Learn and Microsoft Teams allowed the teacher to be aware of her student's progress.

During the second phase of the situation, the students sent the tasks done to the teacher through Microsoft Teams using Casual Learn. As the teacher said: "I got notifications, so I knew that a group was dealing with some tasks" [I]. This made her extend for a few days the duration of the activity, as she realized that several groups could not do it on time. She also mentioned that the information obtained through Microsoft Teams was used for awareness and assessment purposes.

The teacher also said that a Casual Learn dashboard would have been useful for her. With a dashboard, she would see the students' progress at a glance. " $A$ 
dashboard, like the one we have in Moodle makes easier [...] [our work because] it provides a summary [of what is going on]" [I]. Designing and developing such a dashboard is phase of our future work.

Adaptation: this evaluation shows that both teacher and students could adapt the learning process using Casual Learn.

The teacher published in Casual Learn the tasks proposed by two classes before the second phase of the situation started. Thus, she adapted the functionality of Casual Learn to the content of her course. This was done with the support of Casual Learn developers. This process would have been much easier if Casual Learn counted with a task publication interface; in that case, the students would have directly published the tasks in Casual Learn, so she could review them later on. In fact, we have already started working on the development of such a task publication interface for Casual Learn.

Regarding the students, they took advantage of the hundreds of tasks available in Casual Learn and related to Cultural Heritage sites from Valladolid. 13 groups of students completed 30 tasks that were not explicitly related to this learning situation $[\mathrm{L}]$. Thus, they enriched their own learning process taking advantage of the huge number of tasks available in Casual Learn.

Theory: we could see that the pedagogical aims of this learning situation were not transformed, but enriched, by using Casual Learn.

As previously mentioned, the teacher had no previous experience in UL situations. In her view "the time spent with students out of the classroom [school trips] is time to be together, but at the same time to gain knowledge and promote the enthusiasm for acquiring new knowledge. [...]" [I]. This gaining of knowledge is the reason why she organizes school trips every year and she sometimes recommends her students to visit museums or exhibitions related to the course content. In this sense, Casual Learn is an application that offers students these outdoor learning opportunities. The teacher also underlined the alignment of this learning situation to the school's promotion of competence-based learning.

Pragmatism: this evaluation shows that Casual Learn was used by a nonICT expert teacher and her secondary-school students in an authentic collaborative learning situation. Indeed, it was perceived as an easy-to-use application by the teacher [TQ, I] and by the students $[\mathrm{SQ}]$.

The teacher underlined Casual Learn capability to offer content depending on the student's physical location. This is very different from any other tool she had previously used in her classroom: "I have used Moodle and Microsoft Teams in my classroom. But I used them to send homework, to interact with the students, or to send some material. But I have never employed an application that can be used by them, such as Casual Learn, nor any application that can be used directly in the city, nor anything similar" [I].

The teacher also said that orchestrating this learning situation required a significant amount of work, especially during the enactment of its first phase ("[About the amount of hours she spent] A lot, a lot. I cannot quantify them [...] but talking about this specific project, I am thinking about it since the beginning of this course [...] The most intense period was this last month and a half, when I 
was practically working full time for it"). She had to work more than expected, mainly because of the student's lack of critical skills and lack of experience in collaborative learning. Nonetheless, she mentioned that what the students and herself learned from this situation paid her effort: "in this case there is a proper balance [between her effort and the results]. Lot of work is needed, but I understand that this type of methodologies, using new methods, allow to advance in a different and deeper type of learning" [I]. It is noteworthy that the teacher had no previous experience with UL situations, so we can expect that orchestrating similar situations in the future will not take her so much time.

The teacher also considered that the set of tasks contained in Casual Learn is not so well aligned with the content of the course [TQ]. In fact, most of these tasks are related to History of Art [3]. But she understands that they can as well be used for informal learning or touristic purposes ("II would use it] if I go to Salamanca and I have access to this application" [I]). In fact, the students carried out 30 tasks previously existing in Casual Learn.

Synergy: during this evaluation, Casual Learn could be used together with Microsoft Teams, which is widely adopted by Spanish secondary schools.

The integration of Casual Learn and Microsoft Teams was key for the second phase of the situation. The students were used to submit their homework to the teacher through Microsoft Teams [I], so it was natural for them to use this application as a communication channel to send the tasks carried out with Casual Learn. Indeed, the teacher regards Casual Learn as a software tool that she would like to used in the future, together with many others [TQ, I].

\section{Conclusion and future research}

This paper presents a case study that analyzed the orchestration of a collaborative UL situation to learn Cultural Heritage using Casual Learn. This situation involved a teacher and 89 secondary-school students. It included two phases: a first phase in-classroom, where students proposed descriptions of Cultural Heritage sites and tasks to be done when visiting them; and a second phase outclassroom, where students visited the Cultural Heritage sites and carried out the tasks proposed by their colleagues. Thus, this learning situation involved in- and out-classroom activities across physical and virtual spaces.

A non-ICT expert teacher with no previous experience in UL could orchestrate the learning situation without almost any support. The teacher was aware of the students' progress when they were doing in- and out-classroom activities and she could adapt the learning situation accordingly. Specifically, she extended its duration and she further scaffolded her students, mainly due to their lack of critical skills. Orchestrating this UL situation also required a significant effort to the teacher, but she considered it worthy as its pedagogical aims were reached.

Casual Learn was key as an enabling tool for this learning situation, as it bridged in- and out-classroom activities, as well as physical and virtual spaces. In this sense, three functionalities of Casual Learn were especially relevant for this learning situation: the possibility to add tasks and descriptions of Cultural 
Heritage sites, so its functionality can be adapted to the course content; the offering of tasks depending on the learner's physical context, so virtual content is related to physical sites; and its integration to Microsoft Teams, so learners could notify the teacher about out-classroom activities. Finally, Casual Learn offered students additional learning opportunities by suggesting informal learning tasks that some students completed.

The interpretative research paradigm followed in this study makes it difficult to generalize. We will address this caveat by carrying out other similar case studies. We are also further developing Casual Learn according to the suggestions of the participants of this case study: first, we are currently developing a task publication interface; second, we will develop a dashboard, so Casual Learn will offer teachers an overview of the student's progress; third, we will allow the definition of "routes" in Casual Learn, so the application can further guide learners; and fourth, we will develop an iOS version of Casual Learn.

\section{Acknowledgements}

Anonymized

\section{References}

1. Anonymized.

2. Anonymized.

3. Anonymized.

4. D. Abril-López, H. Morón-Monge, M. Morón-Monge, and M. López Carrillo. The learning to learn competence in early childhood preservice teachers: An outdoor and e/m-learning experience in the museum. Future Internet, 13(2):25, 2021.

5. J. Brooke. SUS: a 'quick and dirty' usability scale. In Usability Evaluation in Industry, pages 189-194, London, UK, 1996. Taylor and Francis.

6. E. Ch'ng, Y. Li, S. Cai, and F. Leow. The effects of VR environments on the acceptance, experience, and expectations of Cultural Heritage learning. Journal on Computing and Cultural Heritage, 13(1):Article 7, 2020.

7. L. Cárdenas-Robledo and A. Peña-Ayala. Ubiquitous learning: A systematic review. Telematics and Informatics, 35(5):1097-1132, 2018.

8. C. Delgado-Kloos, D. Hernández-Leo, and J. I. Asensio-Pérez. Technology for learning across physical and virtual spaces. Journal of Universal Computer Science, 18(5):2093-2096, 2012.

9. P. Dillenbourg, M. Nussbaum, Y. Dimitriadis, and J. Roschelle. Design for classroom orchestration. Computers \& Education, 69:485-492, 2013.

10. A. Fermoso, M. Mateos, M. E. Beato, and R. Berjón. Open linked data and mobile devices as e-tourism tools. a practical approach to collaborative e-learning. Computers in Human Behavior, 51:618-626, 2015.

11. J. Greene, B. Kisida, and D. Bowen. The educational value of field trips: taking students to an art museum improves critical thinking skills, and more. Education Next, 14(1):78-86, 2014.

12. E. Guba. Criteria for assessing the trustworthiness of naturalistic inquiries. Educational Technology Research and Development, 1981(29):75, 1981. 
13. G. J. Hwang and C. C. Tsai. Research trends in mobile and ubiquitous learning: A review of publications in selected journals from 2001 to 2010. British Journal of Educational Technology, 42(4):E65-E70, 2011.

14. L. Lucas-Palacios. La enseñanza del patrimonio y de la ciudadanía en las clases de ciencias sociales: un estudio de caso en ESO. PhD thesis, Universidad de Huelva. Departamento de Didáctica de las Ciencias y Filosofía, Huelva, Spain, 2018.

15. M. B. Miles and A. M. Huberman. Qualitative data analysis. An expanded sourcebook. SAGE Publications Inc., Thousand Oaks, California, USA, 1994.

16. M. Milrad, L.-H. Wong, M. Sharples, G.-J. Hwang, and H. Looi, C.-K.and Ogata. Seamless learning: An international perspective on next-generation technologyenhanced learning, pages 95-108. Routledge, Abingdon, UK, 2013.

17. M. Mortara, C. Catalano, F. Belloti, G. Fiucci, M. Houry-Panchetti, and P. Petridis. Learning cultural heritage by serious games. Journal of Cultural Heritage, 15(3):318-325, 2014.

18. J. Muñoz-Cristóbal, I. Jorrín-Abellán, J. Asensio-Pérez, A. Martínez-Monés, L. Prieto, and Y. Dimitriadis. Supporting teacher orchestration in ubiquitous learning environments: A study in primary education. IEEE Transactions on Learning Technologies, 8(1):83-97, 2015.

19. G. Pishtari, M. Rodríguez-Triana, E. Sarmiento-Márquez, M. Pérez-Sanagustín, A. Ruiz-Calleja, P. Santos, L. Prieto, S. Serrano-Iglesias, and T. Väljataga. Learning design and learning analytics in mobile and ubiquitous learning: A systematic review. British Journal of Educational Technology, 51(4):1078-1100, 2020.

20. L. Prieto, M. Dlab, I. Gutiérrez, M. Abdulwahed, and W. Balid. Orchestrating technology enhanced learning: a literature review and conceptual framework. International Journal of Technology Enhanced Learning, 3(6):583-598, 2011.

21. J. Prieto-Pariente. The management of teaching/learning activities under the curricular model of basic competences in the are of technology. $\mathrm{PhD}$ thesis, School of Education and Social Work. University of Valladolid, 2016. In Spanish.

22. T. Ruotsalo, K. Haav, A. Stoyanov, S. Roche, E. Fani, R. Deliai, E. Mäkelä, T. Kauppinen, and E. Hyvönen. Smartmuseum: A mobile recommender system for the web of data. Journal of Web Semantics, 20:50-67, 2013.

23. G. Sonkoly and T. Vahtikari. Innovation in Cultural Heritage: For an Integrated European research policy. Technical report, European Commission, Publications Office (Luxembourg), 2018. Available at: http://openarchive.icomos.org/id/ eprint/2323/1/KI0118044ENN . en\%20\%281\%29.pdf, last visited April 2021. 